\title{
Work-related mental ill health and development of the Surveillance of Occupational Stress and Mental Illness (SOSMI) reporting scheme
}

This paper describes a systematic, voluntary and confidential reporting scheme of new cases of occupational stress and mental illness in the UK (SOSMI). This scheme was designed to gather information on specific types and frequency of ill health seen by consultants within the specialty of psychiatric and psychological medicine. Information obtained from reporting schemes such as SOSMI is crucial in the recognition, understanding and prevention of work-related diseases, as this might come to the attention of specialists long after the patient has left the workplace responsible for the illness.

\section{Background}

There has been an interest in the psychological aspects of work for many years. Since the early part of the 20th century, the Munitions Workers Committee and Industrial Fatigue Board looked at the effects of working hours on employees' health. More recently, there has been an increasing awareness of work-related psychological problems, predominantly anxiety/depression and stress. Results from the 1990 Labour Force Survey (Office of Population Censuses and Surveys, 1992) suggested that 182700 cases of stress or depression were at least partly attributed to work during that year in England and Wales, whereas the 1995 survey of self-reported work-related ill health estimated that 500000 people in Great Britain believed that they were suffering from work-related mental ill health or related illnesses (Jones et al, 1998).

In 1999, the Bristol Stress and Health at Work Study attempted to quantify this problem within the general population. This study was undertaken by postal questionnaire and selected people at random from the electoral register. More than 4000 individuals who responded to the questionnaire were in paid employment at the time of the survey. Results showed that 15-20\% perceived themselves to be 'very or extremely stressed' at work, a further $40-45 \%$ reported 'moderate stress', 25-30\% felt that they were 'mildly stressed' and only about $10 \%$ reported that they were 'not at all' stressed at work (Smith, 2000).

\section{Clinical reporting schemes for occupational disease}

The interaction between psychological ill health and employment is of obvious interest to occupational health practitioners, and since 1996 occupational physicians throughout the UK have reported new cases of workrelated illness to the Occupational Physicians' Reporting Activity (OPRA) surveillance scheme. OPRA is one of a group of clinically-based reporting schemes for occupational disease in the UK and relies on systematic, voluntary and confidential reporting.

Cherry et al (2000) summarised results from the period 1996-1999 of the OPRA surveillance scheme and found that mental ill-health made up $20.9 \%$ of the distribution of cases. A breakdown of diagnoses within the category of mental ill health showed that anxiety and depression contributed more than half $(52.3 \%)$ the cases and work-related stress accounted for a further third (36.4\%).

\section{The SOSMI scheme}

The reporting scheme for occupational disease involving psychiatrists was set up in 1998 and is known as SOSMI. The scheme aims to raise awareness of the effects of work on health, as reported by consultants within the UK. SOSMI benefits from an Advisory Committee that is made up of clinical specialists within the field of mental health, research team members from the Centre for Occupational and Environmental Health at the University of Manchester and representatives from the Health and Safety Executive, a grant from which supports the surveillance schemes.

SOSMI consists of consultants who report either on a monthly basis (core reporters) or those who report for a single randomly assigned month each year (sample reporters). In 2002, there were 15 core and 634 sample reporters in SOSMI, with excellent response rates for both groups (79\% and $77 \%$, respectively). Consultants are asked to report new cases of stress or mental ill 
health which, in their clinical judgement, result from work. Reporters are advised that we are interested in mental illness in all workers, and not only those working within particular industries. The reporting process utilises a card system, and the card content and format was drawn up following detailed discussion with psychiatrists during the pilot phase of the project. Reporting is confidential, and we do not ask for the name of the patient or of the employer.

As a brief example of data produced by SOSMI, in 2002 two-thirds of the cases reported were of anxiety or depression, with the remainder diagnosed as posttraumatic stress disorder, other work-related stress, alcohol or drug misuse and psychotic episodes (as shown in Fig. 1).

The number of cases of psychological ill health reported over this period was highest in teachers, clinical staff (medical and nursing) and police officers. These occupational trends were also reflected in data relating to industries, where the most frequent were health and social work, public administration, and defence and education.

Participants receive a summary report every 3 months of all cases notified to SOSMI by consultants, and additional information from the associated scheme for occupational physicians (OPRA). The summary reports highlight trends in reporting, causal factors and occupational groups or industries of interest. Consultants are encouraged to consider submitting individual cases of educational importance to colleagues, and these might also be included in the quarterly updates. We require informed patient consent before using any clinical case as a 'case of the quarter' and we remove potentially identifiable data from the case summaries to protect patients.

In addition to the regular feedback to reporters, on request we try to answer enquiries and can also provide information on publications which have arisen from the scheme. Further details on SOSMI and the other clinical reporting schemes which comprise The Health and Occupation Reporting network (THOR) are available on the THOR web site (www. coeh.man.ac.uk/thor/).

At the most recent Advisory Committee meeting, the consultant psychiatrists were asked their reasons for participation in the SOSMI reporting scheme; they felt a responsibility to their patients to report health effects relating to work to try to identify hazards and reduce risk to individuals in the workplace, and also that SOSMI data could be used as a bench-marking tool that was highly relevant at a personal level for purposes such as audit and continuing professional development.

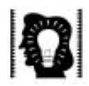

special articles

\section{New developments in SOSMI}

As part of ongoing investigations into the use of medical informatics on reporting of work-related ill health, we carried out a questionnaire survey to gauge attitudes to medical information and technological facilities available to doctors within their day-to-day practice. A total of 380 physicians who report to the THOR schemes (including SOSMI) were selected at random and sent a postal questionnaire. The survey comprised 10 questions involving three main themes: habits and use of electronic means of communication; access to facilities; and willingness to participate in further surveys. An encouraging return rate $(64 \%)$ was obtained, and for each of the survey areas we found very positive responses to the proposed inclusion of medical informatics components within THOR. Initial investigation of data suggested a high comfort level for use of web facilities and e-mail. This was reassuring and will allow us to look at how electronic methods could be developed for effective data collection and dissemination. More detailed analysis is currently underway and will result in further medical informaticsrelated projects. Results from this survey (and future developments) will be quoted on the THOR website as soon as they are available.

An MSc dissertation has been completed that looked at development of a coding system for the precipitating causes of mental ill health reported to the occupational surveillance schemes (Hutton, 2002). The reliability of the coding scheme was tested by observers using data reported to SOSMI and OPRA over a 2-year period, and statistical analysis showed good agreement among observers. The study also looked at the validity of the coding scheme and highlighted difficulties in classification of precipitating events. Further investigation of methodological issues within the reporting schemes is planned, including a review of disease causation or aggravation, and looking at reporting trends.

An additional study is planned to look at differences between reports and diagnoses received from psychiatrists (to SOSMI) and occupational physicians (to OPRA). The use of different diagnostic categories between groups of doctors could be caused by several factors

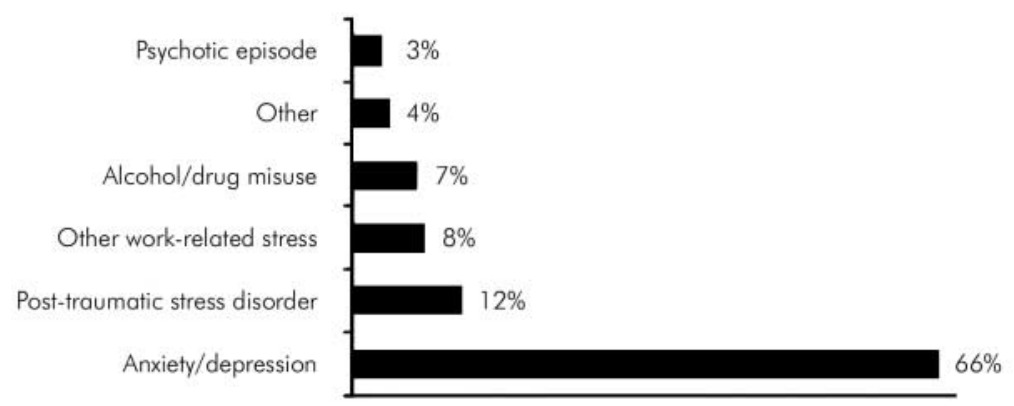

Fig. 1. Estimated number of cases reported to SOSMI by consultants in 2002. 

articles including: case mixes; use of different methodology between schemes and diagnostic preferences associated with clinical practice; experience, and training. The study will involve asking clinicians to write a vignette of a case that they have reported to the schemes, the vignette being sent to a group of scheme reporters, a diagnosis assigned to the case and findings analysed. This study should be useful in the development of future guidance for reporters as a group and at a personal level to allow individual reporters to use this process for audit and continuing personal development purposes.

\section{Acknowledgements}

If you are a reporter to SOSMI we would like to thank you for your support, especially those who sit on the Advisory Committee. However, we are also asking consultants who have not yet been involved in the reporting scheme to assist us. For further information please contact us by phone (0161 275 8490), e-mail (thor@man.ac.uk), or access the website (www.coeh.man.ac.uk/thor/ sosmi.htm). The THOR surveillance schemes, including
SOSMI and OPRA, are supported by a grant from the Health and Safety Executive.

\section{Declaration of interest}

None.

\section{References}

CHERRY, N. M., MEYER, J. D., HOLT, JONES, J. R., HODGSON, J. T., CLEGG, D. L., et al (2000) Surveillance of work- T. A., et al (1998) Self-reported Workrelated diseases by occupational physicians in the UK: OPRA 1996 1999. Occupational Medicine, $\mathbf{5 0}$ 495-503. related Illness in 1995: Results of a Household Survey. Sudbury: HSE Books.

HUTTON, S. (2002) The development of a coding system for causes of mental ill health reported to the SOSMI

Surveillance Programme and an estimation of the validity and reliability of that coding system. MSc Thesis, The OFFICE OF POPULATION CENSUSES AND SURVEYS (1992) Labour Force Survey 1990 and 1991. London: HMSO.

SMITH, A. (2000) The scale of perceived occupational stress. Occupational Medicine, 50, 294-298.

Raymond Agius * *SusanTurner Consultant and Clinical Fellow in Occupational Medicine, Centre for Occupational and Environmental Health, The University of Manchester, Oxford Road, Manchester M13 9PL 